\title{
\begin{tabular}{l|l} 
Mibraries & DSpace@MIT
\end{tabular}
}

\author{
MIT Open Access Articles
}

\section{PROPERTIES OF THE 24 DAY MODULATION IN GX 13+1 FROM NEAR-INFRARED AND X-RAY OBSERVATIONS}

The MIT Faculty has made this article openly available. Please share how this access benefits you. Your story matters.

Citation: Corbet, Robin H. D., Aaron B. Pearlman, Michelle Buxton, and Alan M. Levine. "PROPERTIES OF THE 24 DAY MODULATION IN GX 13+1 FROM NEAR-INFRARED AND XRAY OBSERVATIONS." The Astrophysical Journal 719, no. 1 (July 23, 2010): 979-984. (C) 2010 American Astronomical Society.

As Published: http://dx.doi.org/10.1088/0004-637x/719/1/979

Publisher: Institute of Physics/American Astronomical Society

Persistent URL: http://hdl.handle.net/1721.1/96206

Version: Final published version: final published article, as it appeared in a journal, conference proceedings, or other formally published context

Terms of Use: Article is made available in accordance with the publisher's policy and may be subject to US copyright law. Please refer to the publisher's site for terms of use. 


\title{
PROPERTIES OF THE 24 DAY MODULATION IN GX 13+1 FROM NEAR-INFRARED AND X-RAY OBSERVATIONS
}

\author{
Robin H. D. Corbet ${ }^{1,2}$, Aaron B. Pearlman ${ }^{3}$, Michelle Buxton $^{4}$, and Alan M. Levine ${ }^{5}$ \\ ${ }^{1}$ University of Maryland, Baltimore County, MD, USA; corbet@umbc.edu \\ ${ }^{2}$ CRESST/Mail Code 662, X-ray Astrophysics Laboratory, NASA Goddard Space Flight Center, Greenbelt, MD 20771, USA \\ ${ }^{3}$ Department of Physics, University of Maryland, Baltimore County, 1000 Hilltop Circle, Baltimore, MD 21250, USA; aaronp1@ umbc.edu \\ ${ }^{4}$ Department of Astronomy, Yale University, P.O. Box 208101, New Haven, CT 06520, USA \\ ${ }^{5}$ Kavli Institute for Astrophysics and Space Research, MIT, Cambridge, MA 02139, USA \\ Received 2010 April 13; accepted 2010 June 24; published 2010 July 23
}

\begin{abstract}
A 24 day period for the low-mass X-ray binary (LMXB) GX 13+1 was previously proposed on the basis of seven years of RXTE All-Sky Monitor (ASM) observations and it was suggested that this was the orbital period of the system. This would make it one of the longest known orbital periods for a Galactic LMXB powered by Roche lobe overflow. We present here the results of (1) $K$-band photometry obtained with the SMARTS Consortium CTIO $1.3 \mathrm{~m}$ telescope on 68 nights over a 10 month interval; (2) continued monitoring with the RXTE ASM, analyzed using a semi-weighted power spectrum instead of the data filtering technique previously used; and (3) Swift Burst Alert Telescope (BAT) hard X-ray observations. Modulation near 24 days is seen in both the $K$ band and additional statistically independent ASM X-ray observations. However, the modulation in the ASM is not strictly periodic. The periodicity is also not detected in the Swift BAT observations, but modulation at the same relative level as seen with the ASM cannot be ruled out. If the 24 day period is the orbital period of system, this implies that the X-ray modulation is caused by structure that is not fixed in location. A possible mechanism for the X-ray modulation is the dipping behavior recently reported from XMM-Newton observations.
\end{abstract}

Key words: stars: individual (GX 13+1) - stars: neutron - X-rays: stars

Online-only material: color figures

\section{INTRODUCTION}

GX $13+1$ is a bright low-mass X-ray binary (LMXB, e.g., Homan et al. 2004, and references therein) that has rarely exhibited X-ray bursts (Fleischman 1985; Matsuba et al. 1995), showing that the compact object in the system is a neutron star. An infrared counterpart was identified by Naylor et al. (1991) and Garcia et al. (1992). The infrared counterpart was previously found to vary on timescales of days to tens of days, although no definite orbital period was detected (e.g., Charles \& Naylor 1992; Groot et al. 1996; Bandyopadhyay et al. 2002). From infrared spectroscopy Bandyopadhyay et al. (1999) derived a spectral type of K5 III for the mass-donating star. This classification implies a mass of $5 M_{\odot}$ (Allen 1973), and so the mass donor is the primary star, unlike the majority of LMXBs.

A search was previously made (Corbet 2003; hereafter C03) for periodic modulation in the X-ray flux from GX 13+1 using Rossi X-ray Timing Explorer (RXTE) All-Sky Monitor (ASM) data collected over an interval of almost seven years. From a filtered data set, which excluded measurements with large uncertainties, modulation was found at a period of $24.065 \pm 0.018$ days. The modulation was most clearly detectable at high energies $(5-12 \mathrm{keV})$. Spectral changes were revealed as a modulation in hardness ratio on the 24 day period, and there was a phase shift between the modulation in the 5-12 keV energy band and the $1.5-5 \mathrm{keV}$ band. The high-energy spectrum of GX $13+1$ is unusual in displaying both iron emission and absorption line features, and it was speculated in $\mathrm{C} 03$ that the peculiar spectral and timing properties may be connected. Bandyopadhyay et al. (2002) proposed that the 24 day modulation was part of a long timescale quasi-periodic modulation.

Because of the unusual nature of this modulation, and the somewhat nonstandard technique used to maximize the signal in the ASM data, it was desired to confirm the 24 day period through further observations and determine whether the modulation is present at other wavelengths. We present here both additional RXTE ASM data and also $K$-band observations that confirm the presence of the 24 day period. However, the 24 day period is found not to be strictly periodic in the ASM data. We also analyze Swift BAT observations, which do not show significant modulation near 24 days. We discuss possible causes of the modulation and suggest that it may be caused by dipping.

\section{OBSERVATIONS}

\subsection{X-ray: RXTE ASM}

The RXTE ASM (Levine et al. 1996) consists of three similar Scanning Shadow Cameras (SSCs), sensitive to X-rays in an energy band of approximately $1.5-12 \mathrm{keV}$, which perform sets of 90 s pointed observations ("dwells") so as to cover as much as $\sim 80 \%$ of the sky every $\sim 90$ minutes. Light curves are available in three energy bands: $1.5-3.0 \mathrm{keV}, 3.0-5 \mathrm{keV}$, and 5-12 keV. The Crab produces approximately 75 counts $\mathrm{s}^{-1}$ in the ASM over the entire energy range. Observations of blank field regions away from the Galactic center indicate that background subtraction may produce a systematic uncertainty of about 0.1 counts s ${ }^{-1}$ (Remillard \& Levine 1997). The ASM light curve of GX $13+1$ considered here covers approximately 14 yr (MJD 50,088-55,267; 1996 January 6 to 2010 March 12). For the reasons discussed in Section 3.1, we use ASM light curves binned to 1 day time resolution.

The three SSCs ("1," "2," and "3") that make up the ASM have experienced changes in response with SSC 1 having experienced a gain change of about $10 \%$ per year (A. M. Levine et al. 2010, in preparation). We therefore investigated the ASM light curve of GX 13+1 considering the three SSCs separately. The light 


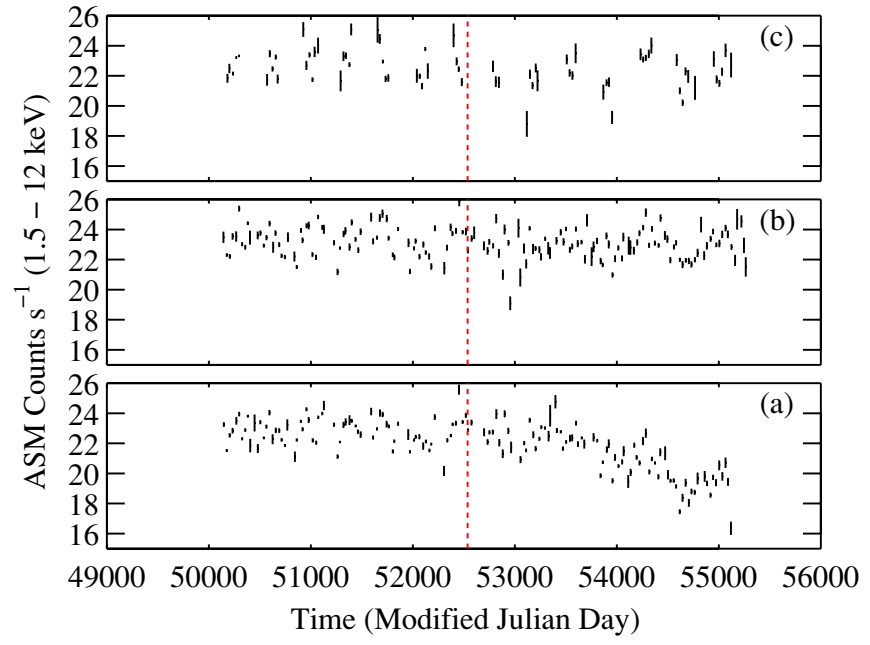

Figure 1. RXTE ASM light curve of GX $13+1$ in 30 day averages. Only time bins which contain a minimum of 20 dwells are plotted. The three panels show data from each SSC separately: (a) SSC 1, (b) SSC 2, and (c) SSC 3. The vertical dashed lines indicate the end of the time range used in C03. SSC 1 shows a flux decline in the second half of the light curve related to gain changes in this detector.

(A color version of this figure is available in the online journal.)

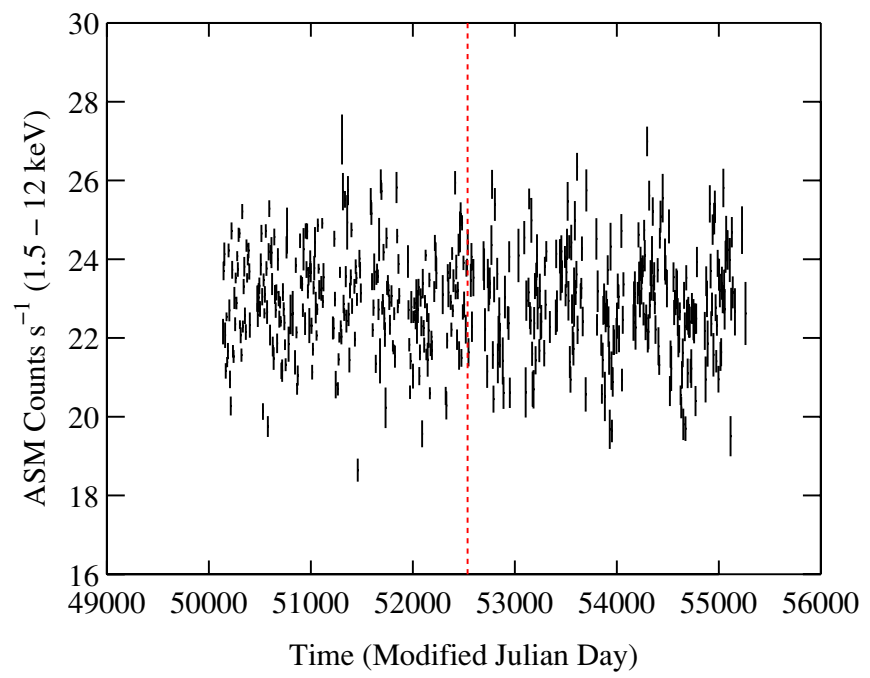

Figure 2. RXTE ASM light curve of GX $13+1$ in 1 week averages. Only time bins that contain a minimum of 20 dwells are plotted. The vertical dashed line indicates the end of the time range used in C03. Only data from SSCs 2 and 3 are included for times after that indicated by the dashed line.

(A color version of this figure is available in the online journal.)

curves obtained in this way are shown in Figure 1. It is seen that although similar count rates are obtained from the three detectors for approximately the first half of the light curve, during the second half an apparent decline in flux occurs only in the light curve from SSC 1. This effect is likely to be due to instrumental changes and we therefore only use SSC 1 data for times before MJD 52,536, i.e., the data range used in C03. The overall RXTE ASM light curve of GX 13+1 obtained with this detector selection is shown in Figure 2. No long-term trend is obvious and the mean flux for the entire ASM energy range is $22.90 \pm 0.01$ (statistical) counts $\mathrm{s}^{-1}$. We also investigated the light curve in the three available energy bands for each SSC. It was found that the energy-separated light curves of SSC 1 show systematic differences from the light curves in the other two SSCs. For this reason, we only use data from SSCs 2 and 3 when examining energy-separated ASM light curves.

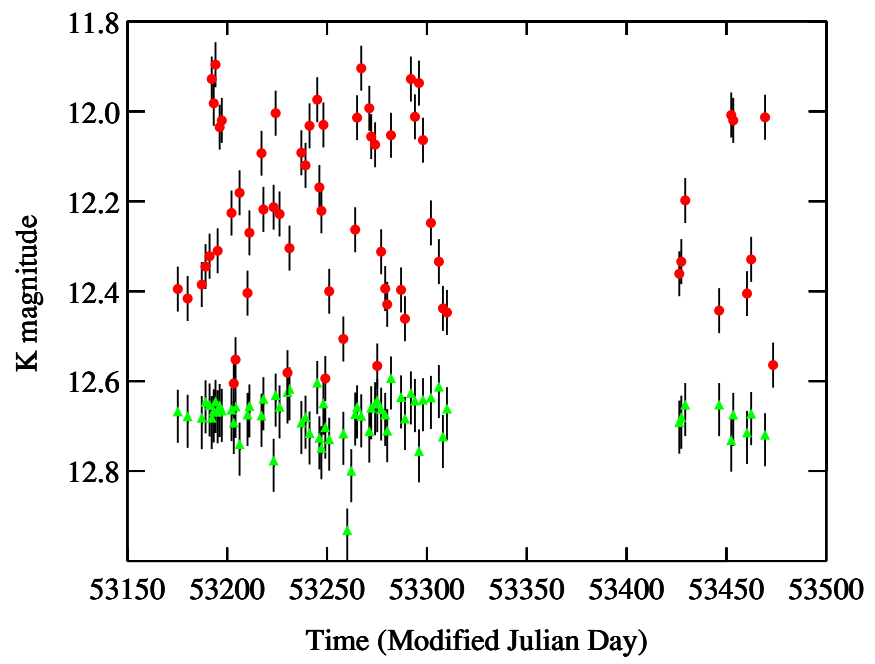

Figure 3. SMARTS CTIO ANDICAM $K$-band photometry of GX $13+1$ (red filled circles) and the comparison star NCL 107 (green filled triangles).

(A color version of this figure is available in the online journal.)

\subsection{Infrared: SMARTS/ANDICAM}

We used the SMARTS Consortium Cerro Tololo InterAmerican Observatory (CTIO) $1.3 \mathrm{~m}$ telescope and ANDICAM detector (DePoy et al. 2003) to obtain 340 infrared images in the $K$ band $(\lambda=2.2 \mu \mathrm{m})$. There were five images taken per day for a total of 68 days over a 10 month interval. The observations span the period from MJD 53,175 (2004 June 19) to 53,473 (2005 April 13). We performed profile-fitting photometry using DAOPHOT after flat fielding the original images with an image constructed from the difference of dome flats obtained with the flat field lamps on and off (Bandyopadhyay et al. 2002). Each set of five infrared images was shifted, aligned, and combined into a final image that was suitable for performing relative photometry. We employed the same star, number 103 of Naylor et al. (1991; "NCL") for all fields to define the profile for each night for the fits. All photometric measurements were also made relative to NCL 103. Conversion to absolute magnitude was made using the mean flux of NCL 106 and the value of $K=$ 12.69 given in Naylor et al. (1991). The standard deviation of the brightness of star NCL 106 is 0.05 mag and we adopt this as an approximation of the uncertainty of the measurements of GX $13+1$. The resulting light curve is plotted in Figure 3 and GX $13+1$ is clearly seen to be highly variable.

\subsection{X-ray: Swift BAT}

The Swift BAT is described in detail by Barthelmy et al. (2005). It is a wide field-of-view instrument that comprises a coded mask aperture with a CdZnTe detector. The data used here come from the $S$ wift/BAT transient monitor results provided by the Swift/BAT team. This provides a light curve covering the energy range $15-50 \mathrm{keV}$. The light curve used here spans the time range of MJD 53,414 (2005 February 13) to 55,267 (2010 March 12) and we rebinned the provided orbital light curve to 1.0 day resolution for our analysis for the reasons discussed in Section 3.1.

\section{ANALYSIS AND RESULTS}

\subsection{Power Spectrum Weighting}

RXTE ASM light curves comprise measurements with a very wide range of error-bar sizes. This means that the error should 


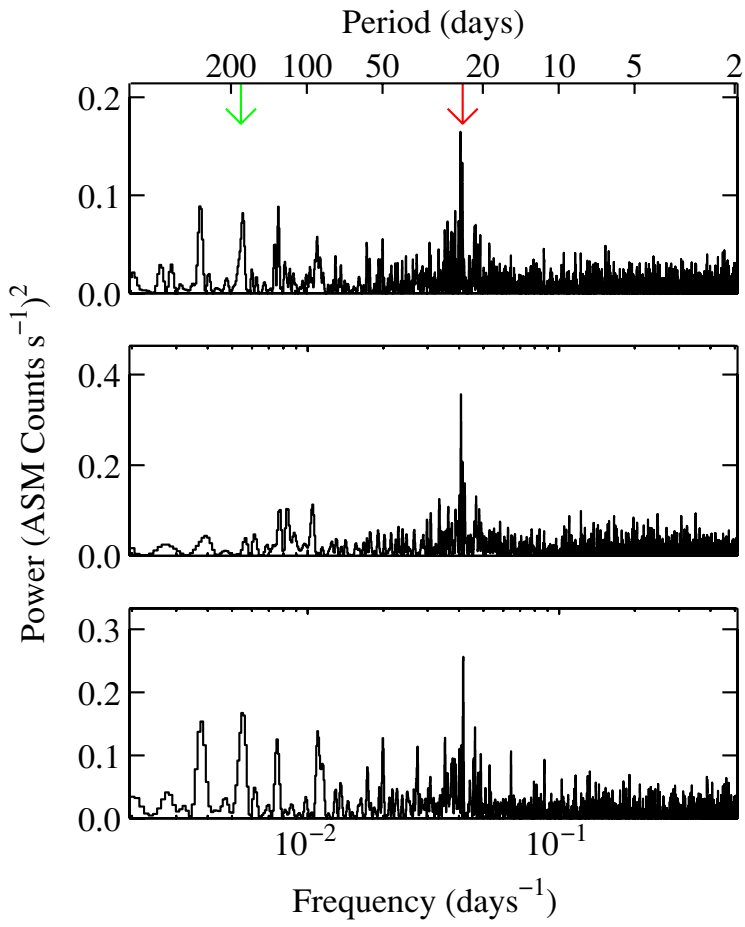

Figure 4. Power spectra of the RXTE ASM observations of GX 13+1. The red arrow marks the period reported in $\mathrm{C} 03$ and the green arrow marks half a year, which is a common artifact in power spectra of ASM light curves. Bottom panel: power spectrum of data presented in $\mathrm{C} 03$; middle panel: power spectrum of data obtained since $\mathrm{C} 03$; top panel: power spectrum of all data.

(A color version of this figure is available in the online journal.)

be taken into account in the calculation of the contribution of each data point to a power spectrum. Scargle (1989) proposed that the effect of unequally weighted data points could be understood by considering the combination of points that coincide. The weighting of a power spectrum is thus analogous to the calculation of a weighted mean. However, as with the weighted mean, in practice the choice of weighting factors needs to be made carefully.

In C03 it was argued that, due to the large-flux variations compared with the error-bar size, simply weighting data points by just the size of their errors was not appropriate and actually decreased the sensitivity of the power spectrum for period detection. A simple data filtering technique was used instead: points with large error bars were excluded, with the threshold chosen to maximize the strength of the 24 day modulation. Although this gave apparently good results, a drawback is that the choice of filtering threshold depends on the assumption that the peak being maximized is indeed a real signal.

In Corbet et al. (2007a), a modified weighting scheme was proposed that could deal with any degree of flux variability compared to error-bar size. This was later noted (Corbet et al. 2007b) to be equivalent to the semi-weighted mean (Cochran 1937, 1954). This technique makes no a priori assumption about the presence of a periodic signal in a data set. The weight chosen for each data point is a combination of both the error bar on that point and the estimated source variability. The source variability is determined by calculating the excess variance of the light curve compared with that predicted from the uncertainties of the data points. Because the semi-weighting scheme does not make any assumptions about the presence of a periodic signal in the data set, and gives appropriate weighting for sources of any brightness, we therefore use semi-weighting in this paper.

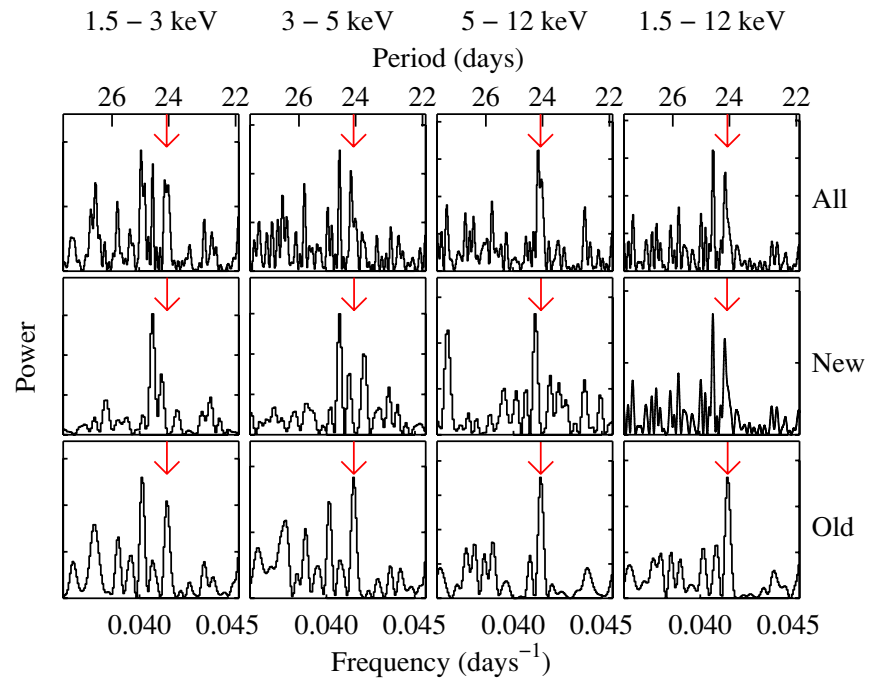

Figure 5. Power spectra of RXTE ASM observations of GX 13+1 separated by time intervals and energy band. Bottom panels: power spectra of data presented in C03; middle panels: power spectra of data obtained since C03; top panels: power spectra of all data. The arrows mark the period reported in $\mathrm{C} 03$. Note that the power spectra are oversampled by a factor of 3 compared to their nominal resolution.

(A color version of this figure is available in the online journal.)

In addition, because the number of ASM and BAT observations per day varies due to orbital precession and other effects for both instruments, we rebinned the light curves to 1 day time resolution to avoid the overweighting of times with larger numbers of dwells that would be effectively introduced otherwise (see, e.g., C03). This overweighting adversely affects power spectra and may introduce artifacts, even if semi-weighting is used. The infrared photometry data have approximately equal error bars and there is thus no benefit to weighting these data. For all the power spectra presented in this paper, we oversample by a factor of 3 compared to the nominal frequency resolution of each power spectrum.

\subsection{RXTE ASM}

We calculated semi-weighted power spectra of the RXTE ASM light curve using three different data selections: (1) the same data used in C03, $6.7 \mathrm{yr}$ duration; (2) only data obtained since $\mathrm{C} 03$, 7.5 yr duration; and (3) the entire ASM light curve, $\sim 14.2 \mathrm{yr}$ duration. These power spectra are shown in Figure 4.

In data set (1) a peak is again found at the proposed 24 day period. In data set (2), which is statistically independent, the strongest peak in the 2-500 day period range is also found near 24 days. In the full data set (3) the 24 day period is the strongest feature in the power spectrum. However, when the peak locations are examined (Figure 5), it can be seen that the peak location is not constant-either between time ranges or among the different energy ranges. It is common to estimate signal coherence using a quality factor, " $Q$," defined as the frequency of a modulation divided by its width in the power spectrum (e.g., van der Klis 2000). However, this simple characterization does not lend itself well to the modulation seen with the ASM in GX 13+1 where, rather than just a broad peak, we see multiple sharp peaks.

We next examined the $1.5-12 \mathrm{keV}$ ASM light curve by dividing up the light curve into several equal length sections and taking the power spectrum of each of the sections. Similar results were obtained for a wide range of number of sections used to divide the light curve, and the results using six sections 


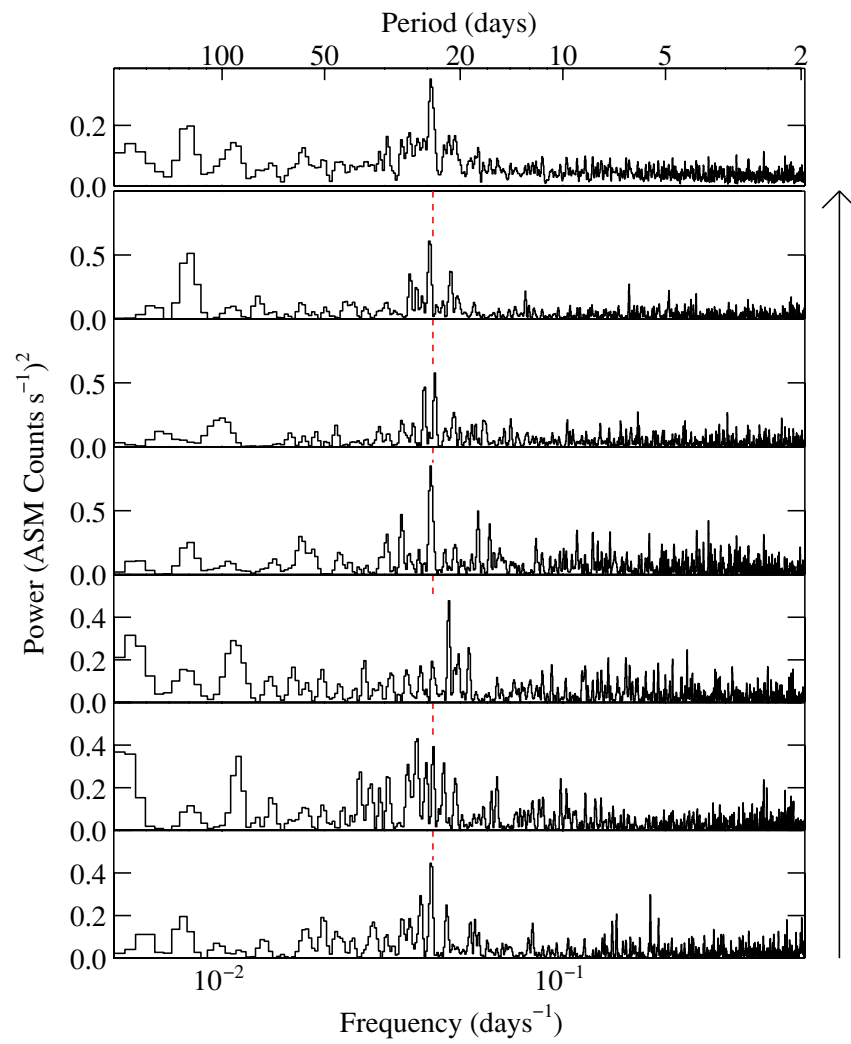

Figure 6. Power spectra of RXTE ASM observations of GX 13+1 divided into six equal time intervals. The top panel shows a non-coherent sum of the power spectra in the six lower panels. The dashed red lines mark the period reported in $\mathrm{C} 03$. The arrow to the right of the figure indicates increasing time.

(A color version of this figure is available in the online journal.)

are plotted in Figure 6. It can be seen that in several of the light-curve sections a peak is present near 24 days, but it is not constant in strength. In the top panel of Figure 6, we also show a simple sum of the power spectra of the individual lightcurve sections (i.e., signal coherence is not considered in adding the power spectra). This shows a prominent peak centered on approximately 24 days. We fitted a Gaussian function to the peak in the light curve and obtained a period of 24.27 days with a formal statistical error of 0.03 days.

\subsection{Infrared}

The $K$-band light curve of GX 13+1 (Figure 3 ) clearly shows variability similar to that seen before in shorter observations. We calculated an unweighted power spectrum of the light curve to search for periodic modulation (Figure 7) and this shows a strong peak near 24 days. The false alarm probability (FAP; Scargle 1982) for finding a peak of this strength anywhere in the entire period range searched of $2-298$ days is $0.5 \%$. The estimated FAP is likely to be somewhat of an underestimate, because the light curve is likely to contain, in addition to periodic modulation, non-periodic components of unknown noise properties. This is seen in optical observations of several LMXBs (e.g., Corbet et al. 1989; Harris et al. 2009). From fitting a sine wave to the light curve we obtain a period of $25.8 \pm 0.3$ days which is similar to, although formally not overlapping, the $24.065 \pm 0.018$ day period reported in $\mathrm{C} 03$. The $K$-band light curve folded on a period of 25.8 days is shown in Figure 8. The mean folded light curve is approximately sinusoidal, however, there is clearly also additional non-periodic variability.

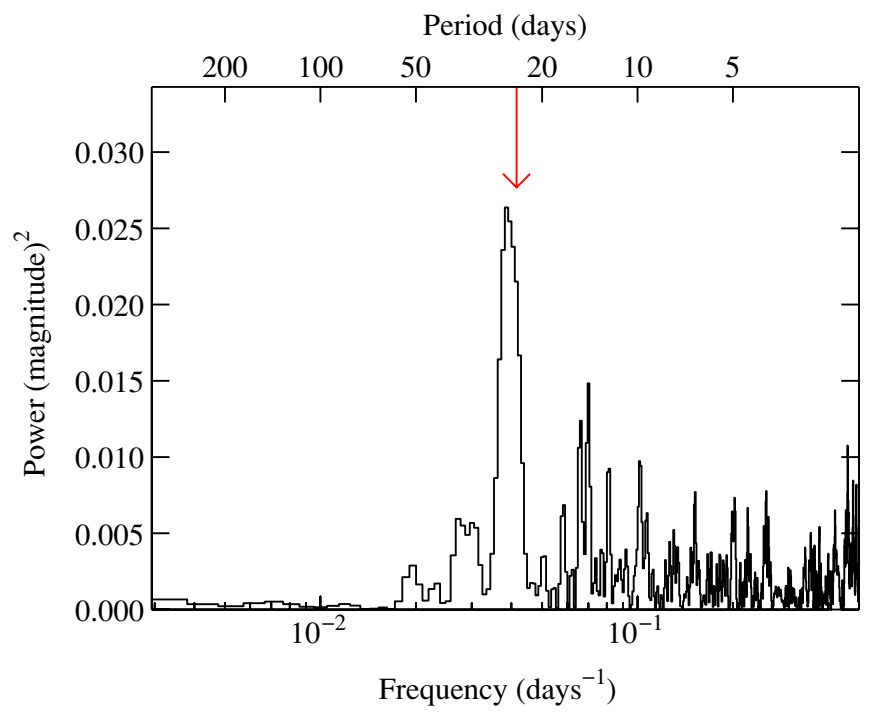

Figure 7. Power spectrum of the SMARTS CTIO ANDICAM $K$-band photometry of GX $13+1$. The red arrow marks the period reported in C03.

(A color version of this figure is available in the online journal.)

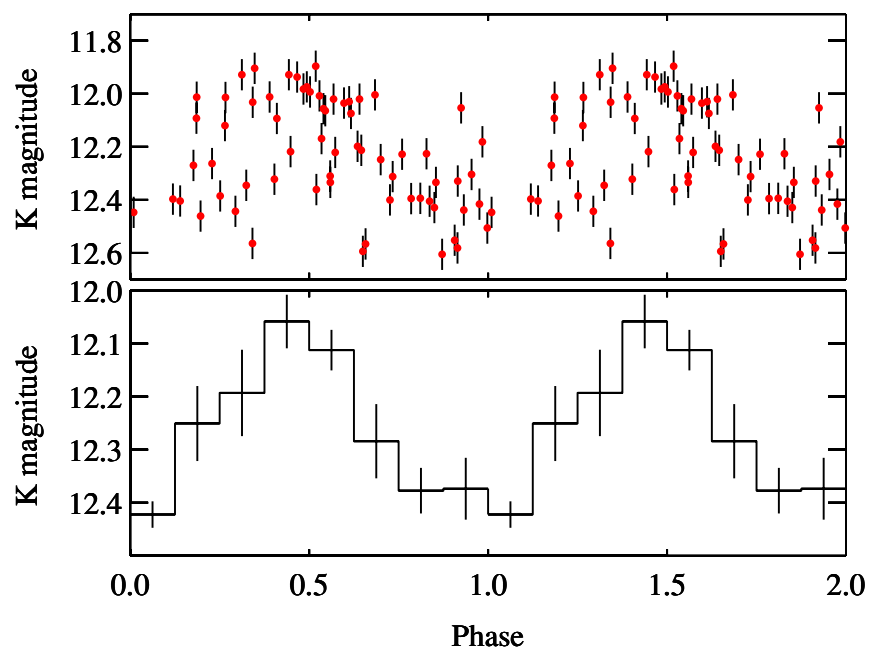

Figure 8. SMARTS CTIO ANDICAM $K$-band observations of GX $13+1$ folded on the strongest peak in the power spectrum at 25.8 days. The two panels show binned and unbinned versions of the light curve.

(A color version of this figure is available in the online journal.)

We investigated whether it was possible to combine our infrared observations with the light curves published by Charles \& Naylor (1992) and Bandyopadhyay et al. (2002) to refine the period measurement. However, the very large intervals between the observations resulted in severe aliasing in the power spectrum which made it impossible to improve the precision of the period determination.

\subsection{Swift BAT}

A power spectrum of the semi-weighted Swift BAT light curve does not show strong modulation at the 24 day period (Figure 9), although there is a small peak of very low significance near the previously reported 24 day period. If we take the small peak near 24 days in the power spectrum of the BAT light curve of GX $13+1$ to be a detection, then the modulation in this energy band (Fourier amplitude divided by mean flux) would be $9 \%$. For comparison, the "incoherent" power spectrum of the ASM full energy band light curve (Figure 6), has an amplitude (Fourier amplitude/mean flux) of $2.4 \%$. Thus, the non-detection 


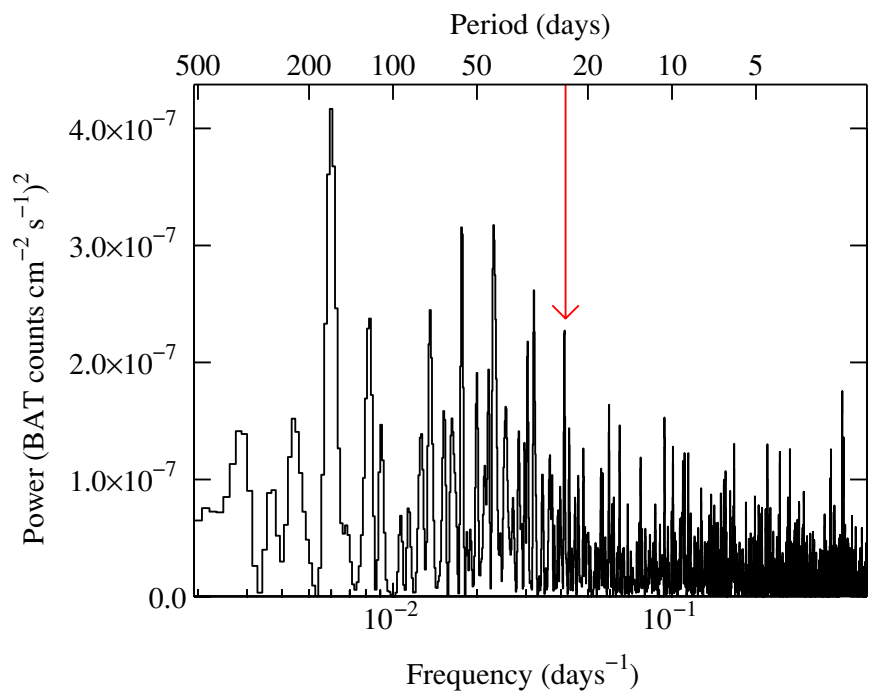

Figure 9. Power spectrum of the Swift BAT light curve of GX 13+1. The red arrow indicates the period reported in $\mathrm{C} 03$.

(A color version of this figure is available in the online journal.)

of modulation with the BAT does not exclude the presence of modulation at the level seen with the ASM.

\section{DISCUSSION}

The previously proposed 24 day period for GX $13+1$ is seen in subsequently obtained, statistically independent, ASM data and is also present in the infrared light curve. The period is also detected in a re-analysis of the initial ASM light curve using a semi-weighted power spectrum. Although the periodicity is not clearly seen in the Swift BAT light curve, the BAT observations appear to have lower sensitivity to a given fractional modulation in GX 13+1 than the ASM observations. A "summary" plot comparing the various power spectra is shown in Figure 10. The multiple peaks in the power spectrum of the ASM light curve (Figure 6) indicate that the X-ray modulation is not strictly periodic and it is therefore difficult to compare the relative phasing of the X-ray and infrared modulations. If a power spectrum of the ASM light curve is calculated using only data obtained during the interval when the infrared observations were made, then no modulation is seen, presumably because the amount of data included does not give sufficient sensitivity to see the modulation. However, a significantly longer stretch of X-ray data cannot be trivially folded: the uncertainty of the period derived from the infrared data alone is too large, and the $\mathrm{X}$-ray data do not provide a unique period to use for folding.

Given the weakness of the X-ray modulation and its lack of coherence it must be considered whether this could be an artifact. However, modulation near 24 days is not seen in other sources with the ASM (e.g., Farrell et al. 2005; Wen et al. 2006). In addition, the modulation seen near this period in the infrared strongly supports an astrophysical rather than an instrumental origin.

In principle, the 24 day modulation could be caused by either an orbital period or a super-orbital period. We note that this period is of the length expected for the orbital period of a Roche lobe-filling K5 III star (Bandyopadhyay et al. 1999). Even though there are uncertainties in the spectral classification, and the radius of the star may differ from that expected from its spectral type due to evolutionary effects caused by mass transfer in the binary, it would be surprising for the expected orbital period to be hugely different from 24 days. In Roche lobe-

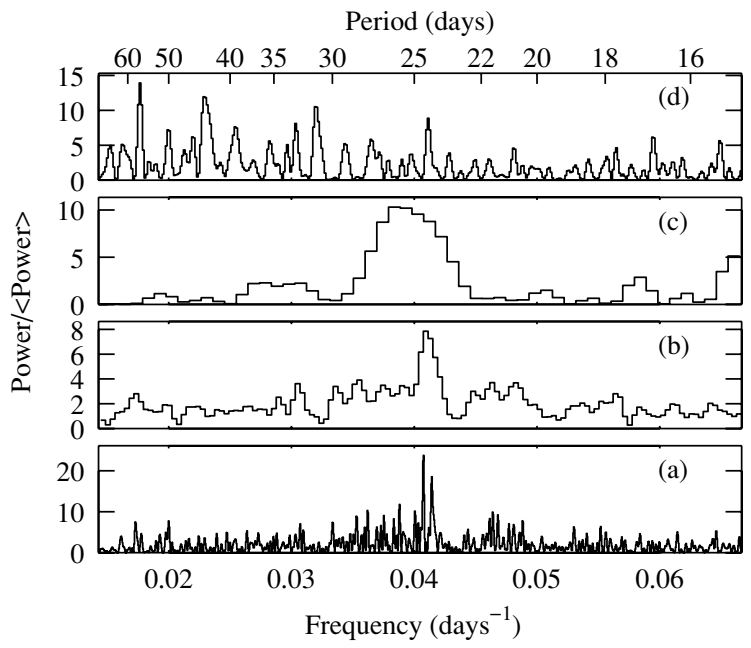

Figure 10. Comparison of the power spectra of GX 13+1 from (a) RXTE ASM (power spectrum of the entire light curve); (b) RXTE ASM (non-coherent sum of power spectra of the separate sections of the ASM light curve shown in Figure 6); (c) $K$-band photometry; (d) Swift BAT. In all cases the power is normalized by the mean power measured over a frequency range of $1 / 200$ to $1 / 2$ day $^{-1}$.

filling systems with super-orbital modulation such as Her X-1, LMC X-1, and SMC X-1, the super-orbital periods are about an order of magnitude longer than the orbital periods (e.g., Charles et al. 2008). In addition, the detection of infrared modulation on only the 24 day period and no other period suggests an orbital origin. An orbital interpretation of the 24 day period is therefore favored for GX 13+1. This orbital period would be one of the longest known for a Galactic LMXB accreting via Roche lobe overflow.

Modulation on the orbital period in the optical and infrared has been seen for a number of LMXBs (e.g., Charles \& Coe 2003). Depending on the X-ray luminosity, the wave band observed, and the system inclination, key contributions to modulation can come from ellipsoidal modulation of the mass donor (which would yield two maxima and minima per orbit), the varying aspect of the heated face of the donor, and eclipses of the accretion disk. Periodic modulation of the X-ray flux of LMXBs on the orbital period is much less commonly observed (e.g., White \& Mason 1985). In systems with high inclination angles eclipses may occur. However, in such systems the central $\mathrm{X}$-ray source is generally obscured by the rim of the accretion disk and X-rays are only observed which are scattered from an accretion disk corona (ADC). At somewhat lower inclination angles, systems might exhibit dips that are thought to be caused by structure at the rim of the accretion disk. Dipping in X-ray binaries is reviewed by, for example, White \& Mason (1985) and Frank et al. (1987).

The dipping behavior reported for GX $13+1$ by M. Díaz Trigo et al. (2010, in preparation) from XMM-Newton observations suggests a possible mechanism for the X-ray modulation in this source. Since the dipping would be expected to be variable in depth and location, this would provide a natural explanation for the lack of strict periodicity found in the ASM light curves. If this dipping interpretation is correct, the location of the material causing the dips could vary and thereby tend to broaden any sharp peaks in a power spectrum. Lower energy X-rays might also be more strongly attenuated by photoelectric absorption leading to a larger orbital modulation. The ASM light curve is only modulated by a few percent on average over the 24 day period and thus, a modest degree of dipping could explain the 
$\mathrm{X}$-ray modulation. If dipping is indeed present, then it would be expected that this should cause modulation on the orbital period. If the 24 day period is not the orbital period of GX $13+1$ then the lack of a detection of the orbital period in the power spectrum would be puzzling. A possible peculiarity, however, is that although the modulation near 24 days is not strictly periodic in X-rays, we do not see any evidence for harmonics of the modulation in the power spectrum. Typically in dipping systems the modulation is non-sinusoidal and so harmonics might be expected to be seen. Another possibility is that structure at the edge of the disk modulates only the portion of the X-rays that comes from an ADC. This might result in modulation that is closer to sinusoidal. However, if the modulation reflects an underlying orbital period, the lack of strict periodicity in an ADC interpretation would be surprising. For example, the ASM light curves of the ADC sources X 1822-371 and X 2127+119 do not show any evidence for period changes (Wen et al. 2006). The infrared modulation would be expected to be caused by one or more of the mechanisms described above (ellipsoidal variations, X-ray heating of the donor, accretion disk eclipses), none of which are as susceptible to modulation phase changes as modulation in the X-ray band such as dipping, which is caused by structure in the outer accretion disk.

It is potentially instructive to compare the variability of GX 13+1 with other LMXBs with long orbital periods. The well-studied LMXB Cygnus X-2 has a relatively long orbital period of 9.8 days (Casares et al. 1998; Elebert et al. 2009). For Cyg X-2, Orosz \& Kuulkers (1999) found that the $B$ and $V$ light curves, when folded on the orbital period, are dominated by ellipsoidal variations and that X-ray heating of the mass donor is relatively unimportant. Orosz \& Kuulkers (1999) suggest that $\mathrm{X}$-ray heating may be unimportant for Cyg X-2 due to a large orbital separation and because a thick accretion disk shields the surface of the mass-donor star. However, in the case of GX 13+1 it seems unlikely that the orbital period is twice the 24 day period as the mass donor would then not fill its Roche lobe, unless the radius of the mass donor is indeed much different from that predicted from its apparent spectral class. Thus, the infrared modulation in GX $13+1$ may be more likely to be caused by $\mathrm{X}$-ray heating of the mass-donating star. For the Galactic black hole candidate systems 1E 1740.7-2942 and GRS 1758-258, periods of 12.7 and 18.5 days, respectively, have been proposed by Smith et al. (2002). The proposed modulation fractions are $3 \%-4 \%$ for both sources and Smith et al. (2002) suggest that the modulations are orbital in origin and that the mass donors are both red giants. These two sources may thus have some similarities with GX 13+1, even though the accreting objects are likely to be black holes rather than neutron stars. GRS 1915+105 has an orbital period of 30.8 days (Neil et al. 2007), comparable to the period of GX 13+1. However, GRS 1915+105 is rather different from GX $13+1$ because it is a microquasar system containing a massive black hole (e.g., Greiner et al. 2001). Long orbital periods have also been reported for extragalactic systems such as the source in M82, which has a 62 day period (Kaaret \& Feng 2007), and NGC 5408 X-1, which has a 115 day period (Strohmayer 2009). However, both of these systems are ultraluminous X-ray sources, probably containing black holes, and so may well also be quite different from GX $13+1$.

\section{CONCLUSION}

A $\sim 24$ day period close to that previously proposed for GX $13+1(\mathrm{C} 03)$ is seen in both new ASM data and near-infrared observations. However, the X-ray modulation is not strictly coherent, which suggests that it may be caused by structure that is not completely phase-locked in the binary system. We propose that the X-ray variability may be caused by dipping behavior. A valuable contribution to an understanding of the system would be a radial velocity orbit of the mass donor and/or the accretion disk from infrared spectroscopy which could show whether the 24 day period is indeed the orbital period, and determine the mass function and also the location of the system components as a function of phase. Additional infrared photometry has the potential to more accurately determine the orbital period.

We thank Tim Naylor for providing us with the photometric data from Charles \& Naylor (1992). We thank Luigi Stella and an anonymous referee for useful comments. This paper made use of Swift/BAT transient monitor results provided by the Swift/ BAT team.

\section{REFERENCES}

Allen, C. W. 1973, Astrophysical Quantities (London: Athlone Press)

Bandyopadhyay, R. M., Charles, P. A., Shahbaz, T., \& Wagner, R. M. 2002, ApJ, 570, 793

Bandyopadhyay, R. M., Shahbaz, T., Charles, P. A., \& Naylor, T. 1999, MNRAS, 306, 417

Barthelmy, S. D., et al. 2005, Space Sci. Rev., 120, 143

Casares, J., Charles, P. A., \& Kuulkers, E. 1998, ApJ, 493, L39

Charles, P., Clarkson, W., Cornelisse, R., \& Shih, C. 2008, New Astron. Rev., 51,768

Charles, P. A., \& Coe, M. J. 2003, in Compact Stellar X-ray Sources, ed. W. H. G. Lewin \& M. van der Klis (Cambridge: Cambridge Univ. Press)

Charles, P. A., \& Naylor, T. 1992, MNRAS, 225, 6P

Cochran, W. G. 1937, Suppl. J. R. Stat. Soc., 4, 102

Cochran, W. G. 1954, Biometrics, 10, 101

Corbet, R. H. D. 2003, ApJ, 595, 1086 (C03)

Corbet, R. H. D., Markwardt, C. B., \& Tueller, J. 2007a, ApJ, 655, 458

Corbet, R. H. D., et al. 1989, MNRAS, 239, 533

Corbet, R. H. D., et al. 2007b, Prog. Theor. Phys. Suppl., 169, 200

DePoy, D. L., et al. 2003, Proc. SPIE, 4841, 827

Elebert, P., Callanan, P. J., Torres, M. A. P., \& Garcia, M. R. 2009, MNRAS, 395, 2029

Farrell, S. A., O’Neill, P. M., \& Sood, R. K. 2005, PASA, 22, 267

Fleischman, J. R. 1985, A\&A, 153, 106

Frank, J., King, A. R., \& Lasota, J.-P. 1987, A\&A, 178, 137

Garcia, M. R., Grindlay, J. E., Bailyn, C. D., Pipher, J. L., Shure, M. A., \& Woodward, C. E. 1992, ApJ, 103, 1325

Greiner, J., Cuby, J. G., \& McCaughrean, M. J. 2001, Nature, 414, 522

Groot, P. J., van der Klis, M., van Paradijs, J., Augusteijn, T., \& Berger 1996, in Cataclysmic Variables and Related Objects, ed. A. Evans \& J. H. Wood (Dordrecht: Kluwer), 367

Harris, R. J., Levine, A. M., Durant, M., Kong, A. K. H., Charles, P., \& Shahbaz, T. 2009, ApJ, 696, 1987

Homan, J., Wijnands, R., Rupen, M. P., Fender, R., Hjellming, R. M., \& di Salvo, T. 2004, A\&A, 418, 255

Kaaret, P., \& Feng, H. 2007, ApJ, 669, 106

Levine, A. M., Bradt, N., Cui, W., Jernigan, J. G., Morgan, E. H., Remillard, R., Shirey, R. E., \& Smith, D. A. 1996, ApJ, 469, L33

Matsuba, E., Dotani, T., Mitsuda, K., Asai, K., Lewin, W. H. G., van Paradijs, J., \& van der Klis, M. 1995, PASJ, 47, 575

Naylor, T., Charles, P. A., \& Longmore, A. J. 1991, MNRAS, 252, 203

Neil, E. T., Bailyn, C. D., \& Cobb, B. E. 2007, ApJ, 657, 409

Orosz, J. A., \& Kuulkers, E. 1999, MNRAS, 305, 1320

Remillard, R. A., \& Levine, A. M. 1997, BAAS, 29, 819

Scargle, J. D. 1982, ApJ, 263, 835

Scargle, J. D. 1989, ApJ, 343, 874

Smith, D. M., Heindl, W. A., \& Swank, J. H. 2002, ApJ, 578, 129

Strohmayer, T. E. 2009, ApJ, 706, 210

van der Klis, M. 2000, ARA\&A, 38, 717

Wen, L., Levine, A. M., Corbet, R. H. D., \& Bradt, H. V. 2006, ApJS, 163 372

White, N. E., \& Mason, K. O. 1985, Space Sci. Rev., 40, 167 